\title{
CORRIGENDUM: ON VINOGRADOV'S MEAN VALUE THEOREM
}

\section{TREVOR D. WOOLEY}

An oversight by the typesetters has led to the omission of the summation condition on the second summation of the first displayed equation on page 392 of [1]. The proof of Lemma 4.1 is consequently very difficult to follow. The expression for $I_{p}(\boldsymbol{\alpha})$ should read

$$
I_{p}(\boldsymbol{\alpha})=\sum_{z=1}^{p^{k}}\left|\sum_{\substack{x \leqslant P \\ x \equiv z\left(\bmod p^{k}\right)}} e\left(\alpha_{1} \Phi_{1}(x)+\ldots+\alpha_{k} \Phi_{k}(x)\right)\right|^{2} .
$$

In addition, we take this opportunity to note that on page 391 , in the two displayed equations following equation (3.15), the summations involving $u_{d+1}$ should read

$$
\sum_{u_{d+1}=1}^{p^{d+1}}
$$

\section{Reference}

1. T. D. Wooley. On Vinogradov's mean value theorem. Mathematika, 39 (1992), 379-399.

Professor T. D. Wooley,

Department of Mathematics,

University of Michigan,

Ann Arbor, MI 48109-1003,

U.S.A.

Received on the 5th of May, 1993. 